\author{
В.М. Журавлев ${ }^{1}$, И.О. Золотовский ${ }^{1}$, Д.А. Коробко ${ }^{1}$, \\ В.В. Светухин ${ }^{1}$, И.О. Явтушенко ${ }^{1}$, М.С. Явтушенко ${ }^{1}$, С.Г. Засканов ${ }^{2}$ \\ ${ }^{1}$ Ульяновский государственный университет, Ульяновск, Россия \\ ${ }^{2}$ Институт систем обработки изображений РАН, Самара, Россия \\ УСИЛЕНИЕ ИНТЕРФЕРЕНЦИОННЫХ СТРУКТУР \\ В СРЕДЕ С НЕЛИНЕЙНОЙ ДИФФУЗИЕЙ
}

\begin{abstract}
Предложена модель усиления периодической структуры, формируемой на поверхности полупроводника под действием лазерного поля. Рассмотрен процесс вырастания поверхностной структуры в среде с нелинейной диффузией дефектов за счет взаимодействия поверхностных плазмон-поляритонов, возбуждаемых на неравновесных электронах, с падающим лазерным излучением. Обнаружен резонансный эффект сверхбыстрого пико- и субпикосекундного усиления генерируемой на поверхности плазмон-поляритонной структуры, при реализации которого может быть получена высококонтрастная решетка дефектов.

Ключевые слова: нелинейная диффузия, поверхностные плазмон-поляритоны, импульсное лазерное облучение, субволновые периодические структуры.
\end{abstract}

\author{
V.M. Zhuravlev', I.O. Zolotovskii', D.A. Korobko', V.V. Svetukhin', \\ I.O. lavtushenko', M.S. lavtushenko', S.G. Zaskanov ${ }^{2}$ \\ ${ }^{1}$ Ulyanovsk State University, Ulyanovsk, Russian Federation \\ ${ }^{2}$ Image Processing Systems Institute of the Russian Academy of Sciences, \\ Samara, Russian Federation
}

\title{
GENERATION AND GROWTH OF INTERFERENCE STRUCTURES IN THE NONLINEAR DIFFUSION MEDIUM
}

We propose a model of radiation induced generation of periodic structure on the semiconductor surface. Here, the surface structure generation and growth in a medium with the nonlinear diffusion defects is considered. This process is due to interaction between the surface plasmon polaritons excited on nonequilibrium electrons and the incident laser radiation. A resonance ultra-fast pico- and subpicosecond growth of plasmon-polariton structure generated on the surface has been observed. A defect grating of high-contrast can be obtained through this process.

Keywords: nonlinear diffusion, surface plasmon polaritons, pulsed laser irradiation, subwavelength periodic structures. 


\section{Введение}

Образование на поверхности пространственно-модулированных структур с периодом как порядка длины световой волны [1-4], так и значительно меньшей [5-9], - одно из самых распространенных явлений, происходящих при воздействии лазерного излучения на конденсированные среды. Возникновение на поверхности полупроводников периодических структур с большой контрастностью, значительной амплитудой рельефа и периодом, значительно меньшим длины волны облучающего лазера [10-13], представляет особый интерес.

Лазерное излучение позволяет, благодаря высокой плотности потока фотонов, реализовать процессы многоквантового поглощения света, поэтому в случае фоточувствительных материалов (например, кремния) на поверхности полупроводника происходит генерация значительного числа неравновесных носителей, скорость которой является нелинейной функцией интенсивности [1-3].

При облучении ультракороткими импульсами (длительностью менее 100 фс) время электрон-фононной релаксации материала, как правило, значительно превышает время воздействия импульса тр, поэтому кристаллическая решетка остается «холодной» и практически не принимает участия в поглощении лазерного излучения. Последнее поглощается электронной подрешеткой и вызывает образование на поверхности полупроводника тонкой пленки металлизации [3-5]. Процесс такой металлизации поверхности полупроводника является нетермическим фазовым переходом, инициированным световым излучением. Если под действием лазерного излучения на поверхности или в объеме полупроводника формируется металлизированный слой, то при выполнении определенного условия на границе «металлизированная поверхность - среда» возможна реализация условий для возбуждения на неравновесных электронах поверхностных плазмонполяритонов (ПП), взаимодействующих с падающим лазерным излучением. В результате этого взаимодействия на поверхности облучаемого материала формируется периодическая структура, модулированная световым полем, с периодом порядка либо значительно меньшим центральной длины волны источника [14-16].

В ряде случаев может наблюдаться исключительно высокая контрастность образующихся периодических структур, которая представляет отдельный интерес и не может быть объяснена, с нашей точки 
зрения, действием только периодически модулированного интерференционного поля лазерного излучения.

В настоящей работе для объяснения эффекта генерации высококонтрастных структур с относительно большой амплитудой рельефа предлагается модель, связанная с нелинейной зависимостью коэффициента диффузии от концентрации неравновесных носителей (или в ряде случаев дефектов различных типов, возникающих на поверхности полупроводника).

В общем случае такого рода модели исследовались достаточно давно, особенно в связи с задачами распространения тепла в средах с нелинейными коэффициентами теплопроводности [17-19], которые представляют собой прямой аналог диффузионных уравнений для концентраций дефектов.

В рассматриваемой нами задаче коэффициент диффузии связан с концентрацией диффундирующей примеси, дефектов или неравновесных носителей с помощью следующей формулы:

$$
D=\frac{D_{0}}{n+n_{0}},
$$

где $D_{0}=D_{p} n_{0}, D_{0}$ и $n_{0}-$ некоторые постоянные; $n$ - концентрация изучаемых дефектов; $D_{p}-$ собственно диффузии, $\mathrm{cm}^{2} / \mathrm{c}$.

Уменьшение коэффициента диффузии объясняется возможной кластеризацией условных дефектов на поверхности облучаемого материала. Свойства уравнений с таким коэффициентом диффузии исследовались в работах [19-22]. Одним из важных свойств этих уравнений является наличие у них богатого семейства точных решений, что позволяет исследовать поведение системы, опираясь не на численный анализ, а на свойства точных решений при тех или иных начальных и граничных условиях. Таким образом, можно без привлечения численного счета предъявлять точные аргументы аналитических решений в форме периодических функций, а также применять различные методы возмущений и асимптотического анализа.

\section{1. Уравнение нелинейной диффузии}

Используя коэффициент диффузии (1), можно записать уравнение диффузии дефектов в следующем виде: 


$$
\frac{\partial n}{\partial t}=\frac{\partial}{\partial z}\left(\frac{D_{0}}{n+n_{0}} \cdot \frac{\partial n}{\partial z}\right)-\mu n+G,
$$

где $t$ - время; $z$ - координата; $\mu$ - скорость рекомбинации дефектов; $G-$ скорость генерации дефектов.

Вводя функцию $\xi=n+n_{0}$, преобразуем это уравнение к следующему виду:

$$
\frac{\partial \xi}{\partial t}=\frac{\partial}{\partial z}\left(\frac{D_{0}}{\xi} \cdot \frac{\partial \xi}{\partial z}\right)-\mu \xi+G_{\text {ef }} .
$$

Здесь введен эффективный коэффициент генерации дефектов

$$
G_{\text {ef }}=G+\mu n_{0} .
$$

Далее, переходя к нормированной координате $x=z / \sqrt{D_{0}}$, получаем уравнение

$$
\frac{\partial \xi}{\partial t}=\frac{\partial^{2} \ln \xi}{\partial x^{2}}-\mu \xi+G_{\text {ef }}
$$

\section{2. Отрицательная эффективная диффузия. Диффузионный «взрыв»}

В рассматриваемой нами модели быстрое формирование интерференционной картины происходит за счет взаимодействия падающей волны с образующимися ПП [23-29]. Характерные времена составляют значения порядка субпикосекунд, а характерные длины соответствуют глубине проникновения излучения и периоду плазмон-поляритонной структуры, т.е. в общем случае менее 1 мкм $(\mu \mathrm{m})$. Поскольку диффузионная длина существенно больше характерных длин, то можно предполагать, что, наряду с быстрыми процессами (формирования интерференционного поля падающего лазерного импульса и ПП), в самой среде происходят относительно медленные процессы формирования структур - образование контрастного рельефа на поверхности. Для выявления условий возникновения длинноволновых возмущений за счет тех или иных неустойчивостей можно воспользоваться методом многомасштабных разложений [17-19], позволяющим выявить растущие при данных условиях моды. 
Для анализа полученного уравнения (4) используем метод решения уравнения в медленных переменных. Это означает, что, наряду со стандартными единичными масштабами времени, ответственными за переходные процессы в системе, решение уравнения (4) будет содержать зависимость от медленных переменных времени $T=\varepsilon t$ и длины $X=\sqrt{\varepsilon} x[17,18]$. Здесь $\varepsilon-$ безразмерный малый параметр, характеризующий время возникновения структуры по отношению к масштабу времени $\tau$. При этом безразмерный масштаб длины возникающей структуры определяется малой величиной $\sqrt{\varepsilon}$. Зависимость функции $\xi$ от координат и времени можно записать в следующем виде:

$$
\xi=\xi(x, t)=\chi(t, X, T, \varepsilon) .
$$

Представим функцию $\xi(x, t)$ в виде ряда по малому параметру $\varepsilon$ :

$$
\chi(t, X, T, \varepsilon)=\xi_{0}(t, X, T)+\xi_{1}(t, X, T) \varepsilon+O\left(\varepsilon^{2}\right) .
$$

С использованием стандартных для метода многомасштабных разложений соотношений

$$
\frac{\partial \xi}{\partial t}=\frac{\partial \chi}{\partial t}+\varepsilon \frac{\partial \chi}{\partial T}, \frac{\partial \xi}{\partial x}=\sqrt{\varepsilon} \frac{\partial \chi}{\partial X}
$$

уравнение (4) преобразуется к виду

$$
\chi_{t}=-\mu \chi+G_{\text {ef }}-\varepsilon \frac{\partial \chi}{\partial T}+\varepsilon \frac{\partial^{2} \chi}{\partial X^{2}} .
$$

Коэффициенты разложения (6) удовлетворяют следующим уравнениям:

$$
\xi_{0, t}=-\mu \xi_{0}+g, \quad \xi_{1, t}=-\mu \xi_{1}-\frac{\partial \xi_{0}}{\partial T}+\frac{\partial^{2} \ln \xi_{0}}{\partial X^{2}} .
$$

Решение в нулевом порядке принимает вид

$$
\xi_{0}=C(X, T) e^{-\mu t}+G_{\mathrm{ef}} / \mu,
$$

где $C(X, T)$ - постоянная интегрирования, зависящая в данном случае от медленных переменных. Аналогично решение в первом порядке можно представить как

$$
\xi_{1}=C_{1}(X, T) e^{-\mu t}+e^{-\mu t} \int_{0}^{t} e^{\mu t^{\prime}}\left[-\frac{\partial \xi_{0}}{\partial T}+\frac{\partial^{2} \ln \xi_{0}}{\partial X^{2}}\right] d t^{\prime},
$$


где $C_{1}(X, T)$ - постоянная интегрирования, также зависящая от медленных переменных.

Для нахождения решения в первом порядке вычисляем интегралы в (11):

$$
\begin{gathered}
I_{1}=-\int_{0}^{t} e^{\mu t^{\prime}} \frac{\partial \xi_{0}}{\partial T} d t^{\prime}=-\frac{\partial C}{\partial T} t \\
I_{2}=\int_{0}^{t} e^{\mu t^{\prime}} \frac{\partial^{2} \ln \xi_{0}}{\partial X^{2}} d t^{\prime}=\int_{0}^{t} e^{\mu t^{\prime}} \frac{\left(C e^{-\mu t^{\prime}}+G_{\mathrm{ef}}\right) C_{X X} e^{-\mu t^{\prime}}-C_{X}^{2} e^{-2 \mu t^{\prime}}}{\left(C e^{-\mu t^{\prime}}+G_{\mathrm{ef}} / \mu\right)^{2}} d t^{\prime}= \\
=C_{X X} \frac{\mu}{G_{\mathrm{ef}}}\left(t+\frac{1}{\mu} \ln \left(C e^{-\mu t}+G_{\mathrm{ef}} / \mu\right)\right)-\frac{C_{X}^{2}}{\mu C\left(C e^{-\mu t}+G_{\mathrm{ef}} / \mu\right)} .
\end{gathered}
$$

В (13) введены обозначения: $C_{X}=\partial C / \partial X$ и $C_{X X}=\partial^{2} C / \partial X^{2}$. Интегралы $I_{1}$ и $I_{2}$ содержат слагаемые, растущие в асимптотике при $t \rightarrow \infty$ по линейному закону. Согласно стандартной процедуре исключения резонансов необходимо потребовать, чтобы эти слагаемые обратились в ноль. В интеграле $I_{2}$ при $t \rightarrow \infty$ слагаемые, содержащие множитель $t$, взаимно сокращаются. Поэтому функция $C(X, T)$, определяющая динамику поля дефектов, удовлетворяет уравнению

$$
\frac{\partial C}{\partial T}-\frac{\mu}{G_{\text {ef }}} \frac{\partial^{2} C}{\partial X^{2}}=0 .
$$

Таким образом, получено уравнение, определяющее динамику генерируемых неравновесных зарядов (дефектов) в поле излучения, которое можно назвать дифффузионным уравнением с эффективной диффузией: $D_{\text {ef }}=\mu / G_{\text {ef }}$.

Самым интересным следствием уравнения (14) является возможность «возникновения» отрицательной эффективной диффузии, когда $\mu / G_{\mathrm{ef}}<0$. Режим отрицательной диффузии может быть реализован в двух случаях:

$$
\left.\mu<0 \text { и } G_{\mathrm{ef}}>0 \text { (т.е. } G+\mu n_{0}>0\right)
$$

или

$$
\left.\mu>0 \text { и } G_{\mathrm{ef}}<0 \text { (т.е. } G+\mu n_{0}<0\right) \text {. }
$$


Второй случай, по всей видимости, соответствует быстрой рекомбинации соответствующих дефектов (неравновесных носителей), вплоть до нулевого значения за ограниченный промежуток времени, и, скорее всего, не может представлять существенного интереса для разного рода практических применений.

Очевидно, что наибольший интерес для нас будет представлять первый случай, соответствующий экспоненциально нарастающей генерации дефектов.

Уравнение (14) представляет собой параболическое уравнение в медленных переменных с эффективным коэффициентом диффузии, равным $\mu / G_{\text {ef }}$, который меняет знак в зависимости от знака $G$. Пространственно-периодические решения являются суперпозицией решений вида [30]

$$
C(X, T)=e^{p T}(A \cos (k X)+B \sin (k X)),
$$

где параметр $p$ связан с волновым числом $k$ по медленной координате $X$ соотношением $p=-\frac{k^{2} \mu}{G_{\text {ef }}}$.

Подставляя (16) в (14) и возвращаясь в «исходные координаты» времени и длины, находим:

$$
\begin{gathered}
C(x, t)=e^{(p \varepsilon-\mu) t}(A \cos (k \sqrt{\varepsilon} x)+B \sin (k \sqrt{\varepsilon} x))= \\
=e^{g t}(A \cos (q z)+B \sin (q z)),
\end{gathered}
$$

где $q=2 \pi / d ; d$ - пространственный период неоднородности, определяемый в случае формирования ПП начальными условиями возникновения неоднородности и составляющий $d \approx 0,01-10$ мкм $[1,29]$.

Для эффективного инкремента усиления периодических возмущений при отрицательной эффективной диффузии верно соотношение

$$
g=p \varepsilon-\mu=-\frac{q^{2} \mu D_{0}}{G_{\text {ef }}}-\mu=-\mu\left(1+\frac{q^{2} D_{0}}{G_{\text {ef }}}\right)=-\mu\left(1+\frac{q^{2} n_{0} D_{p}}{G_{\text {ef }}}\right)=-\mu+g_{D} .
$$

Приведем оценку характерных масштабов длины и времени для различных процессов в рассматриваемой нелинейной системе.

Для задач, связанных с радиационным облучением, параметры в системе могут быть выбраны в следующем виде: $D_{p}=10^{-2}-10^{2} \mathrm{~cm}^{2} / \mathrm{c}$, 
$n_{0} \gg 10^{21} \mathrm{~cm}^{-3}, G=10^{26}-10^{34} \mathrm{~cm}^{-3} / \mathrm{c}^{-1}[1,23]$. Тогда для скорости фотогенерации свободных носителей с хорошей степенью точности подходит соотношение [1,23]

$$
G \approx \alpha I / \hbar \omega,
$$

где $I$ - интенсивность света используемого лазерного источника; $\alpha-$ линейный коэффициент поглощения полупроводника, который обеспечивает генерацию неравновесных зарядов и изменяется в исключительно широком интервале значений в зависимости от длины волны лазерного источника (таблица).

Так, для кристаллического кремния имеются следующие значения параметров в зависимости от длин волн лазерного излучения [23]. Для коэффициента рекомбинации можно записать соотношение

$$
\mu \cong 1 / \tau_{c}-\sigma_{c} I / \hbar \omega,
$$

где $\tau_{c}$ - время рекомбинации (релаксации) зарядов, составляющее от 1 мкс до 1 пс; $\sigma_{c}$ - сечение поглощения на отдельном носителе, составляющее порядка $10^{-16}-10^{-18} \mathrm{~cm}^{2}$.

Параметры поглощения кремния при облучении различными лазерными источниками

\begin{tabular}{|l|c|c|c|c|}
\hline Длина волны излучения, мкм & 0,265 & 0,53 & 1,06 & 10,6 \\
\hline Параметр поглощения $d=\alpha^{-1}$ & 6 нм & 500 нм & 200 мкм & 1 мм \\
\hline
\end{tabular}

Таким образом, скорость линейной рекомбинации неравновесных зарядов $\mu$ определяется разностью величины скорости генерации неравновесных носителей на поверхности полупроводниковой структуры за вычетом параметра, характеризующего скорость отрицательной рекомбинации, определяемой эффектом локального усиления поля на дефектах, а в нашем случае - на генерируемых неравновесных носителях.

Как видно из соотношений (15), усиление диффузионной неустойчивости происходит в том случае, если коэффициент рекомбинации становится отрицательным:

$$
\mu \cong 1 / \tau_{c}-\sigma_{c} I / \hbar \omega<0,
$$


но при этом эффективный коэффициент генерации неравновесных носителей остается положительным:

$$
G_{\mathrm{ef}} \cong \alpha I / \hbar \omega+n_{0}\left(1 / \tau_{c}-\sigma_{c} I / \hbar \omega\right)>0 .
$$

При этом в случае, когда интенсивность лазерного излучения стремится к значению

$$
I_{c} \cong \frac{\hbar \omega n_{0}}{\tau_{c}} \cdot \frac{1}{n_{0} \sigma_{c}-\alpha},
$$

реализуется режим резонансного «сверхбыстрого» усиления (при $\left.\sigma_{c} n_{0}>\alpha\right)$, когда параметр $\left|G_{\mathrm{ef}}\right| \rightarrow 0$ и, следовательно, параметр, связанный с диффузионным усилением, достигают огромных значений $\left|g_{D}\right| \rightarrow \infty$. Кроме того, критическое значение интенсивности является значением, вблизи которого поведение системы может качественно изменяться.

С учетом характерных значений соответствующих параметров значение резонансной интенсивности можно оценить в интервале значений $10^{6}-10^{10} \mathrm{~B} / \mathrm{cm}^{2}$, т.е. на уровне мощностей, обеспечиваемых умеренно мощными доступными лазерными источниками.

Так, при $I<I_{c}$, но при $I>\hbar \omega / \sigma_{c} \tau_{c}$, имеет место диффузионное усиление поля неоднородных дефектов - неравновесных зарядов. В области $\left|I_{c}-I\right| \rightarrow 0$ (но при $I<I_{c}$ ) наблюдается исключительно мощное усиление неоднородного «рельефного» поля неравновесных носителей. А при $I>I_{c}$ происходит сильное диффузионное «затухание» неоднородного поля дефектов. В области $\left|I_{c}-I\right| \rightarrow 0$ (но $I>I_{c}$ ) имеет место сверхбыстрая диссипация неоднородного (периодического) поля дефектов - неравновесных носителей.

Видно, что в области критической интенсивности $I_{c}$ динамика системы может кардинальным образом изменяться при незначительном изменении мощности лазерного источника. Ясно, что в общем случае для анализа поведения системы в этой узкой области значений интенсивности излучения необходимо учитывать нелинейные эффекты более высокого порядка (прежде всего влияние Оже-рекомбинации, имеющей кубическую зависимость от концентрации неравновесных зарядов [23]). 
Из соотношения (18) следует, что усиление генерируемой периодической структуры тем сильнее, чем меньше период наведенной на поверхности плазмон-поляритонной структуры (ППС). Заметим, что в первом приближении период ППС, возникающей между полупроводником и наведенной пленкой металлизации, приблизительно равен толщине последней $[11,23]$. Толщину фотоиндуцированной пленки металлизации по порядку величины можно считать обратно пропорциональной величине эффективного поглощения полупроводника, т.е. с хорошей степенью точности можно считать $q \approx \alpha$.

Тогда решение (18) можно переписать в виде

$$
g \approx-\left(\frac{1}{\tau_{c}}-\frac{\sigma_{c} I}{\hbar \omega}\right)\left(1+\frac{\alpha^{2} n_{0} D_{p}}{\frac{\alpha I}{\hbar \omega}+n_{0}\left(\frac{1}{\tau_{c}}-\frac{\sigma_{c} I}{\hbar \omega}\right)}\right) .
$$

При этом видно, что наибольшего усиления периодически модулированной плотности неравновесных зарядов (и, как следствие, высоты рельефа поверхности облучаемого полупроводника) можно добиться в условиях больших значений $\alpha$, а также больших значений произведения $n_{0} \sigma_{c}$. При этом, согласно (15.1), должно выполняться условие $G_{\text {ef }}>0$, и в идеальном случае $G_{\text {ef }} \Rightarrow 0$. Исходя из этого (см. таблицу), можно сделать заключение о желательности использования лазерных комплексов, работающих в оптическом и ближнем УФ-диапазонах, для которых (в случае кремниевых структур) величина параметра $\alpha$ достигает огромных значений - свыше $10^{8} \mathrm{M}^{-1}$.

На рисунке представлены зависимости $g(I)$ (кривые 1-4), построенные на основе соотношения (24) с параметрами среды и излучения, подобранными таким образом, чтобы выполнялось условие (15.1). Из рисунка видно, что при определенных условиях приведенные зависимости могут носить резонансный характер.

Отдельно следует отметить, что в условиях $n_{0} \rightarrow \infty$, когда нелинейностью можно пренебречь, диффузионная составляющая инкремента усиления стремится к значению $g_{D} \cong-q^{2} D_{p}$ и всегда остается отрицательной, что способствует скорейшему затуханию соответствующих интерференционных компонент - возмущений, что особенно 
актуально в условиях больших значений коэффициента диффузии. Вместе с тем скорость генерации поверхностной дефектной структуры может достигать гигантских (особенно в условиях резонанса, когда $G_{\mathrm{ef}} \rightarrow 0$ ) значений $\left(g_{D}=10^{11}-10^{14} \mathrm{c}^{-1}\right)$, и здесь можно говорить о диффузионном «взрыве», приводящем к сверхбыстрому образованию высококонтрастной периодической структуры.

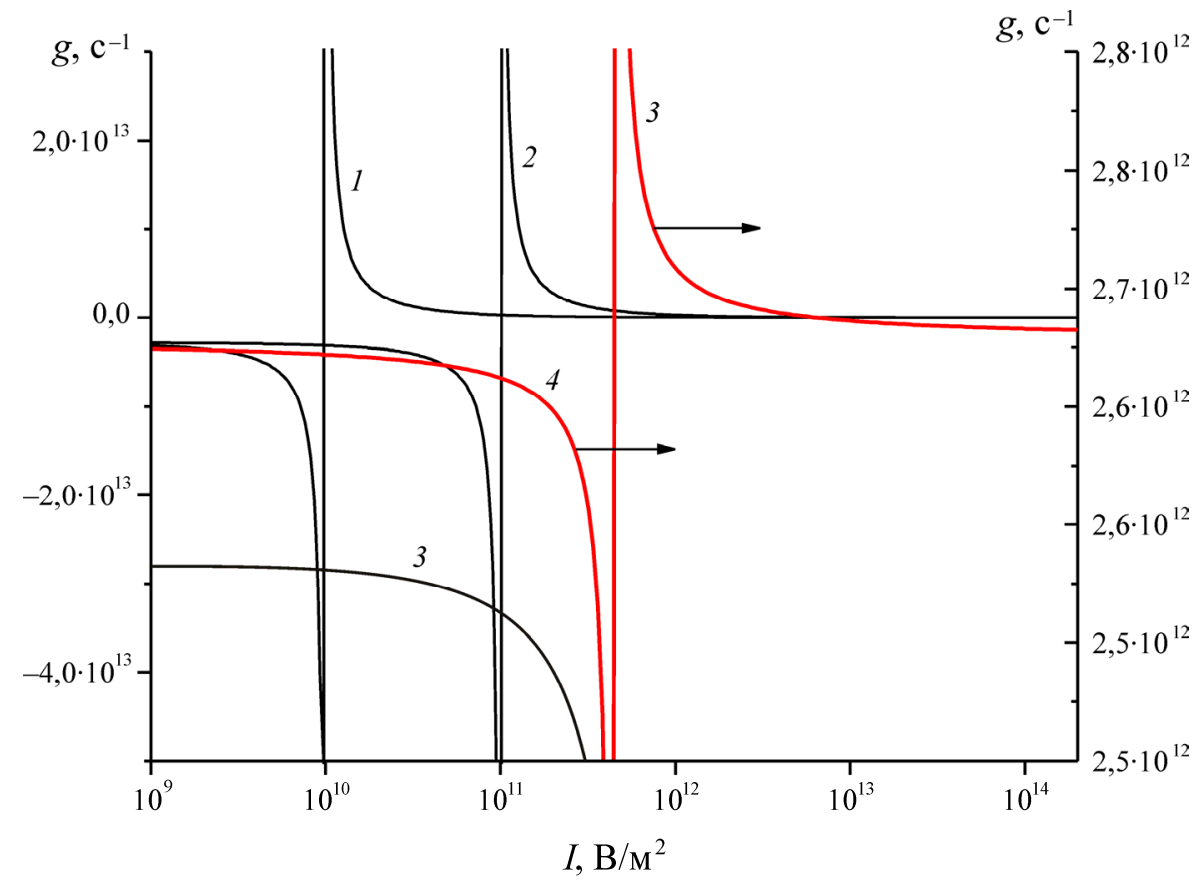

Рис. Зависимость эффективного инкремента усиления от интенсивности лазера $g(I)$ для различных параметров среды и излучения: $1-\tau_{c}=10^{-7}$ с; $\lambda=265$ нм; $\alpha=(6 \mathrm{нм})^{-1} ; n_{0}=0,168 \cdot 10^{29} ; \quad D_{p}=10^{-4} ; \sigma_{c}=10^{-20} \mathrm{~m}^{2} ; 2-\tau_{c}=10^{-7}$ c; $\lambda=265$ нм; $\alpha=(6 \mathrm{нм})^{-1} ; n_{0}=0,168 \cdot 10^{29} ; D_{p}=10^{-4} ; \sigma_{c}=10^{-20} \mathrm{~m}^{2} ; 3-\tau_{c}=10^{-6} \mathrm{c} ; \lambda=265$ нм; $\alpha=\left(6 \mathrm{HM}^{-1} ; n_{0}=10^{30} ; \quad D_{p}=10^{-3} ; \quad \sigma_{c}=10^{-20} \mathrm{M}^{2} ; 4-\tau_{c}=10^{-7} \mathrm{c} ; \lambda=530\right.$ нм; $\alpha=$ $=(500 \mathrm{HM})^{-1} ; n_{0}=2 \cdot 10^{27} ; D_{p}=10^{-6} ; \sigma_{c}=10^{-19} \mathrm{M}^{2}$

\section{Заключение}

В рамках предложенной модели нелинейной диффузии обнаружен сильный резонансный эффект, способный приводить к сверхбыстрому пико- и субпикосекундному усилению генерируемой на поверхности плазмон-поляритонной структуры. В этом случае может быть 
получена высококонтрастная решетка дефектов, разрастающаяся за время, значительно меньшее пикосекунды. Процесс образования высококонтрастной периодической структуры можно представить следующим образом [1, 23]:

1. Процесс начинается с возникновения периодически модулированного интерференционного светового поля падающего лазерного излучения и ПП.

2. В периодически модулированном по интенсивности световом поле происходит усиливаемый диффузионной неустойчивостью локальный нагрев поверхности.

3. При достаточной мощности лазерного излучения неоднородный нагрев вызывает неоднородное плавление и сверхбыстрый вынос вещества, при этом происходит «запоминание» интерференционного рельефа.

Одновременно с этим реализуется сценарий возникновения неустойчивости за счет положительной обратной связи по следующей схеме. Появление микрорельефа поверхности с определенными периодом и фазой способствует повышенному поглощению в пиковых позициях структуры, что еще более увеличивает глубину модуляции температуры и приводит к дальнейшему повышению поглощения и т.д. При этом время генерации высококонтрастной периодической структуры с большой амплитудой неоднородности может быть значительно меньше 1 пс. Представляется, что именно диффузионная неустойчивость в системах с нелинейной диффузией может быть механизмом, обеспечивающим «запись» высококонтрастных периодических структур рельефа микро- и нанометрового масштабов.

Работа выполнена при поддержке Министерства образования и науки Российской Федераџии (проект № 14.Z50.31.0015), а также при частичной финансовой поддержке Российского фонда фундаментальных исследований (проект № 14-32-50507).

\section{Список литературы}

1. Воздействие мощного лазерного излучения на поверхность полупроводников и металлов: нелинейно-оптические эффекты и нелинейно-оптическая диагностика / С.А. Ахманов, В.И. Емельянов, 
Н.И. Коротеев, В.Н. Семиногов // Успехи физических наук. - 1985. T. 147. - С. 675-745.

2. Емельянов В.И., Рогачева А.В. Зажигание и распространение уединенной волны образования точечных дефектов при интенсивной лазерной генерации электрон-дырочных пар в полупроводниках и диэлектриках // Квантовая электроника. - 1998. - Т. 25, № 11. - С. 1017-1022.

3. Surface electromagnetic waves in optics / A.M. Bonch-Bruevich, M.N. Libenson, V.S. Makin, V.V. Trubaev // Optical Engineering. - 1992. Vol. 31, № 4. - P. 716-730.

4. Емельянов В.И., Панин И.М. Образование нанометровых упорядоченных дефектно-деформационных структур в твердых телах при воздействии на них потоков энергии // Физика твердого тела. - 2009. Т. 39, вып. 11. - С. 2029-2035.

5. Теория образования ансамбля нанокластеров на поверхности кристаллов $\mathrm{CdTe}$ при одноимпульсном лазерном воздействии / В.И. Емельянов, А. Байдуллаева, А.И. Власенко, П.Е. Мозоль // Квантовая электроника. - 2008. - Т. 38, № 3. - С. 245-250.

6. Интерференция поверхностных электромагнитных волн и периодические структуры, образующиеся при воздействии интенсивного света на поверхность полупроводника / В.В. Баженов, А.М. БончБруевич, М.Н. Либенсон, В.С. Макин // Письма в журнал технической физики. - 1984. - Т. 10, вып. 24. - С. 1520-1526.

7. Фемтосекундная лазерная запись субволновых одномерных квазипериодических наноструктур / Е.В. Голосов, В.И. Емельянов, А.А. Ионин, Ю.Р. Колобов, С.И. Кудряшов, А.Е. Лигачев, Ю.И. Новоселов, Л.В. Селезнев, Д.В. Синицин // Письма в журнал экспериментальной и теоретической физики. - 2009. - Т. 90, вып. 2. - С. 116-120.

8. Mechanism for self-formation of periodic grating structures on a metal surface by a femtosecond pulse / S. Sakabe, M. Hashida, S. Tokita, S. Namba, K. Okamura // Phys. Rev. B. - 2009. - Vol. 79. - P. 033409.

9. Ripple formation during deep hole drilling in copper with ultrashort laser pulses / A. Welch, T.H.R. Crawford, D.S. Wilkinson, H.K. Haugen, J.S. Preston // Appl. Phys. A. - 2007. - Vol. 89. - P. 1001.

10. Поверхностные плазмон-поляритонные моды и наноструктурирование полупроводников фемтосекундными лазерными импульсами / В.С. Макин, Ю.И. Пестов, Р.С. Макин, А.Я. Воробьев // Оптический журнал. - 2009. - Т. 76, № 9. - С. 38-44. 
11. Bonse J., Munz M., Sturm H. Structure formation on the surface of indium phosphide irradiated by femtosecond laser pulses // J. Appl. Phys. 2005. - Vol. 97. - P. 013538.

12. Subsurface modification in indium phosphide induced by single and multiplefemtosecond laser pulses: a study of the formation of periodic ripples / M.G. Couillard, A. Borowiec, H.K. Haugen, J.S. Preston, E.M. Griswold, G.A. Botton // J. Appl. Phys. - 2007. - Vol. 101. - P. 033519.

13. Borowiec A., Haugen H.K. Subwavelength ripple formation on the surface of compound semiconductors irradiated with femtosecond laser pulses // Appl. Phys. Lett. - 2003. - Vol. 42. - P. 4462-4464.

14. Фемтосекундное наноструктурирование кремниевых поверхностей / С.В. Заботнов, Л.А.Головань, И.А. Остапенко [и др.] // Письма в журнал экспериментальной и теоретической физики. - 2006. - Т. 83, № 2. - C. 76-79.

15. Vorobyev A.Y., Makin V.S., Guo C. Periodic ordering of random surface femtosecond laser pulses on metals // J. Appl. Phys. - 2007. Vol. 101. - P. 034903.

16. Berini P., De Leon I. Surface plasmon-polariton amplifiers and lasers // Nature Photonics. - 2012. - Vol. 6, № 1. - P. 16-24.

17. Самарский А.А., Михайлов А.П. Математическое моделирование. Идеи. Методы. Примеры. - М.: Физматлит, 2001. - 320 с.

18. Режимы с обострением в задачах для квазилинейных параболических уравнений / А.А. Самарский, В.А. Галактионов, С.П. Курдюмов, А.П. Михайлов. - М.: Наука, 1987. - 477 с.

19. Аристов С.Н. Периодические и локализованные точные решения уравнения $h_{t}=\Delta \ln h / /$ Прикладная механика и техническая физика. - 1999. - Т. 40, № 1. - С. 22-26.

20. Журавлев В.М. Об одном классе моделей автоволн в активных средах с диффузией, допускающих точные решения // Письма в журнал экспериментальной и теоретической физики. - 1997. - Т. 65, вып. 3. - С. 285-290.

21. Журавлев В.М. Диффузионные цепочки Тоды в моделях нелинейных волн в активных средах // Журнал электронной и теоретической физики. - 1998. - Т. 114, вып. 5. - С. 1897-1914.

22. Журавлев В.М. Точные решения уравнений нелинейной диффузии в двумерном координатном пространстве // Теоретическая и математическая физика. - 2000. - Т. 124, № 2. - С. 265-278. 
23. Коротеев Н.И., Шумай И.Л. Физика мощного лазерного излучения. - М.: Наука, 1991. - 312 с.

24. Климов В.В. Наноплазмоника. - М.: Физматлит, 2009. - 480 с.

25. Поверхностные поляритоны. Электромагнитные волны на поверхностях и границах раздела сред / под ред. В.М. Аграновича, Д.Л. Миллса. - М.: Наука, 1985. - 526 с.

26. Либенсон М.Н., Бонч-Бруевич А.М., Макин В.С. Поверхностные поляритоны и силовое действие излучения // Успехи физических наук. - 1988. - Т. 155. - С. 719-721.

27. Возбуждение поверхностных и волноводных мод интенсивным лазерным излучением и их влияние на характер поверхностного разрушения конденсированных сред / А.М. Бонч-Бруевич, М.К. Коченгина, М.Н. Либенсон, В.С. Макин, С.Д. Пудков, В.В. Трубаев // Известия АН СССР. Сер.: Физическая. - 1982. - Т. 46, № 6. - С. 1185-1195.

28. Surface electromagnetic waves in optics. / A.M. Bonch-Bruevich, M.N. Libenson, V.S. Makin, V.V. Trubaev // Optical Engineering. - 1992. Vol. 31, № 4. - P. 718-730.

29. Макин В.С., Макин Р.С. Основы взаимодействия ультракороткого лазерного излучения с конденсированными средами: монография. - Димитровград, 2013. - 236 с.

30. Тихонов А.Н., Самарский А.А. Уравнения математической физики. - М.: Наука, 1972. - 73 с.

\section{References}

1. Akhmanov S.A., Emel'ianov V.I., Koroteev N.I., Seminogov V.N. Vozdeistvie moshchnogo lazernogo izlucheniia na poverkhnost' poluprovodnikov i metallov: nelineino-opticheskie effekty i nelineino-opticheskaia diagnostika [Exposure of high-power laser radiation on the surface of semiconductors and metals: nonlinear optical effects and nonlinear-optical diagnostics]. Uspekhi fizicheskikh nauk, 1985, vol. 147, pp. 675-745.

2. Emel'ianov V.I., Rogacheva A.V. Zazhiganie i rasprostranenie uedinennoi volny obrazovaniia tochechnykh defektov pri intensivnoi lazernoi generatsii elektron-dyrochnykh par v poluprovodnikakh i dielektrikakh [Ignition and propagation of a solitary wave of the formation of point defects of intense laser generation of electron-hole pairs in semiconductors and dielectrics]. Kvantovaia elektronika, 1998, vol. 25, no. 11, pp. 1017-1022. 
3. Bonch-Bruevich A.M., Libenson M.N., Makin V.S., Trubaev V.V. Surface electromagnetic waves in optics. Optical Engineering, 1992, vol. 31, no. 4, pp. 716-730.

4. Emel'ianov V.I., Panin I.M. Obrazovanie nanometrovykh uporiadochennykh defektno-deformatsionnykh struktur $\mathrm{v}$ tverdykh telakh pri vozdeistvii na nikh potokov energii [Formation of ordered nanometer defect-deformation structures in solids when exposed to energy flows]. Fizika tverdogo tela, 2009, vol. 39, iss. 11, pp. 2029-2035.

5. Emel'ianov V.I., Baidullaeva A., Vlasenko A.I., Mozol' P.E. Teoriia obrazovaniia ansamblia nanoklasterov na poverkhnosti kristallov CdTe pri odnoimpul'snom lazernom vozdeistvii [The theory of the formation of an ensemble of nanoclusters on the surface of CdTe crystals with one pulse laser irradiation]. Kvantovaia elektronika, 2008, vol. 38, no. 3, pp. 245-250.

6. Bazhenov V.V., Bonch-Bruevich A.M., Libenson M.N., Makin V.S. Interferentsiia poverkhnostnykh elektromagnitnykh voln i periodicheskie struktury, obrazuiushchiesia pri vozdeistvii intensivnogo sveta na poverkhnost' poluprovodnika [Interference surface electromagnetic waves and periodic structures formed when exposed to intense light to the surface of the semiconductor]. Pis'ma v zhurnal tekhnicheskoi fiziki, 1984, vol. 10, iss. 24, pp. 1520-1526.

7. Golosov E.V., Emel'ianov V.I., Ionin A.A., Kolobov Iu.R., Kudriashov S.I., Ligachev A.E., Novoselov Iu.I., Seleznev L.V., Sinitsin D.V. Femtosekundnaia lazernaia zapis' subvolnovykh odnomernykh kvaziperiodicheskikh nanostruktur [Femtosecond laser writing of one-dimensional quasi-periodic subwavelength nanostructures]. Pis'ma v zhurnal eksperimental'noi i teoreticheskoi fiziki, 2009, vol. 90, iss. 2, pp. 116-120.

8. Sakabe S., Hashida M., Tokita S., Namba S., Okamura K. Mechanism for self-formation of periodic grating structures on a metal surface by a femtosecond pulse. Journal of Applied Physics B, 2009, vol. 79, p. 033409.

9. Welch A., Crawford T.H.R., Wilkinson D.S., Haugen H.K., Preston J.S. Ripple formation during deep hole drilling in copper with ultrashort laser pulses. Journal of Applied Physics A, 2007, vol. 89, p. 1001.

10. Makin V.S., Pestov Iu.I., Makin R.S., Vorob'ev A.Ia. Poverkhnostnye plazmon-poliaritonnye mody i nanostrukturirovanie poluprovodnikov femtosekundnymi lazernymi impul'sami [Surface plasmonpolariton modes and nanostructured semiconductors by femtosecond laser pulses]. Opticheskii zhurnal, 2009, vol. 76, no. 9, pp. 38-44. 
11. Bonse J., Munz M., Sturm H. Structure formation on the surface of indium phosphide irradiated by femtosecond laser pulses. Journal of Applied Physics, 2005, vol. 97, p. 013538.

12. Couillard M.G., Borowiec A., Haugen H.K., Preston J.S., Griswold E.M., Botton G.A. Subsurface modification in indium phosphide induced by single and multiplefemtosecond laser pulses: a study of the formation of periodic ripples. Journal of Applied Physics, 2007, vol. 101, p. 033519.

13. Borowiec A., Haugen H.K. Subwavelength ripple formation on the surface of compound semiconductors irradiated with femtosecond laser pulses. Applied Physics Letters, 2003, vol. 42, pp. 4462-4464.

14. Zabotnov C.V., Golovan' L.A., Ostapenko I.A. [et al.] Femtosekundnoe nanostrukturirovanie kremnievykh poverkhnostei [Femtosecond nanostructuring of silicon surfaces]. Pis'ma $v$ zhurnal elektronnoi $i$ teoreticheskoi fiziki, 2006, vol. 83, no. 2, pp. 76-79.

15. Vorobiev A.Y., Makin V.S., Guo C. Periodic ordering of random surface femtosecond laser pulses on metals. Journal of Applied Physics, 2007, vol. 101, p. 034903.

16. Berini P., De Leon I. Surface plasmon-polariton amplifiers and lasers. Nature Photonics, 2012, vol. 6, no. 1, pp. 16-24.

17. Samarskii A.A., Mikhailov A.P. Matematicheskoe modelirovanie. Idei. Metody. Primery [Math modeling. Ideas. Methods. Examples]. Moscow, 2001. 320 p.

18. Samarskii A.A., Galaktionov V.A., Kurdiumov S.P., Mikhailov A.P. Rezhimy s obostreniem v zadachakh dlia kvazilineinykh parabolicheskikh uravnenii [Modes with peaking in problems for quasi-linear parabolic equations]. Moscow, 1987. $477 \mathrm{p}$.

19. Aristov S.N. Periodicheskie i lokalizovannye tochnye resheniia uravneniia $h_{t}=\Delta \ln h$ [Recurrent localized and accurate solutions of the equation]. Prikladnaia mekhanika i tekhnicheskaia fizika, 1999, vol. 40, no. 1, pp. 22-26.

20. Zhuravlev V.M. Ob odnom klasse modelei avtovoln v aktivnykh sredakh s diffuziei, dopuskaiushchikh tochnye resheniia [About a class of autowaves models in active media with diffusion, allowing exact solutions]. Pis'ma v zhurnal elektronnoi i teoreticheskoi fiziki, 1997, vol. 65, iss. 3, pp. 285-290. 
21. Zhuravlev V.M. Diffuzionnye tsepochki Tody $\mathrm{v}$ modeliakh nelineinykh voln $\mathrm{v}$ aktivnykh sredakh [Diffusion Toda models of nonlinear waves in active media]. Zhurnal elektronnoi i teoreticheskoi fiziki, 1998, vol. 114, iss. 5, pp. 1897-1914.

22. Zhuravlev V.M. Tochnye resheniia uravnenii nelineinoi diffuzii $v$ dvumernom koordinatnom prostranstve [Exact solutions of the nonlinear diffusion equations in the two-dimensional coordinate space]. Teoreticheskaia i matematicheskaia fizika, 2000, vol. 124, no. 2, pp. 265-278.

23. Koroteev N.I., Shumai I.L. Fizika moshchnogo lazernogo izlucheniia [The physics of high-power laser radiation]. Moscow, 1991. 312 p.

24. Klimov V.V. Nanoplazmonika [Nanoplasmonics]. Moscow, 2009. $480 \mathrm{p}$.

25. Poverkhnostnye poliaritony. Elektromagnitnye volny na poverkhnostiakh i granitsakh razdela sred [Surface polaritons. Electromagnetic waves on the surfaces and Interfaces]. Ed. by V.M. Agranovich, D.L. Mills. Moscow, 1985. 526 p.

26. Libenson M.N., Bonch-Bruevich A.M., Makin V.S. Poverkhnostnye poliaritony i silovoe deistvie izlucheniia [Surface polaritons and intense laser radiation]. Uspekhi fizicheskikh nauk, 1988, vol. 155, pp. 719-721.

27. Bonch-Bruevich A.M., Kochengina M.K., Libenson M.N., Makin V.S., Pudkov S.D., Trubaev V.V. Vozbuzhdenie poverkhnostnykh i volnovodnykh mod intensivnym lazernym izlucheniem i ikh vliianie na kharakter poverkhnostnogo razrusheniia kondensirovannykh sred [Excitation of surface waveguide modes and intense laser radiation and their influence on the nature of the surface destruction of condensed matter]. Izvestiia akademii nauk SSSR. Seriia Fizicheskaia, 1982, vol. 46, no. 6, pp. 1185-1195.

28. Bonch-Bruevich A.M., Libenson M.N., Makin V.S., Trubaev V.V. Surface electromagnetic waves in optics. Optical Engineering, 1992, vol. 31, no. 4, pp. 718-730.

29. Makin V.S., Makin R.S. Osnovy vzaimodeistviia ul'trakorotkogo lazernogo izlucheniia s kondensirovannymi sredami [Fundamentals of interaction of ultrashort laser radiation with condensed media]. Dimitrovgrad, 2013. $236 \mathrm{p}$.

30. Tikhonov A.N., Samarskii A.A. Uravneniia matematicheskoi fiziki [Equations of mathematical physics]. Moscow, 1972. $73 \mathrm{p}$.

Получено 15.12.2015. 